\title{
Sparse and Skew Hashing of K-Mers
}

\section{Giulio Ermanno Pibiri ${ }^{1}$}

\author{
${ }^{1}$ ISTI-CNR, Pisa, Italy
}

\begin{abstract}
Motivation: A dictionary of $k$-mers is a data structure that stores a set of $n$ distinct $k$-mers and supports membership queries. This data structure is at the hearth of many important tasks in computational biology. High-throughput sequencing of DNA can produce very large $k$-mer sets, in the size of billions of strings in such cases, the memory consumption and query efficiency of the data structure is a concrete challenge. Results: To tackle this problem, we describe a compressed and associative dictionary for $k$-mers, that is: a data structure where strings are represented in compact form and each of them is associated to a unique integer identifier in the range $[0, n)$. We show that some statistical properties of $k$-mer minimizers can be exploited by minimal perfect hashing to substantially improve the space/time trade-off of the dictionary compared to the best-known solutions.
\end{abstract}

Availability: The $\mathrm{C}++_{+}$implementation of the dictionary is available at https://github.com/jermp/sshash.

Contact: giulio.ermanno.pibiri@isti.cnr.it

\section{Introduction}

A $k$-mer is a string of length $k$ over the DNA alphabet $\{\mathrm{A}, \mathrm{C}, \mathrm{G}, \mathrm{T}\}$. Software tools based on $k$-mers are in widespread use in Bioinformatics. Many large-scale analyses of DNA share the elementary need of determining the exact membership of $k$-mers to a given set $\mathcal{S}$, i.e., they rely on the space/time efficiency of a dictionary data structure for $k$ mers [Chikhi et al., 2021]. This work proposes such an efficient dictionary. More precisely, the problem we study here is defined as follows. Given a large string over the DNA alphabet (e.g., a genome or a pan-genome), let $\mathcal{S}$ be the set of all its distinct $k$-mers, with $|\mathcal{S}|=n$. A dictionary for $\mathcal{S}$ is a data structure that supports the following two operations:

- for any $k$-mer $g, \operatorname{Lookup}(g)$ returns a unique integer $0 \leq i<n$ if $g \in \mathcal{S}$ (the "identifier" of $g$ ) or $i=-1$ if $g \notin \mathcal{S}$;

- for any $0 \leq i<n$, Access $(i)$ extracts the $k$-mer $g$ for which $\operatorname{Lookup}(g)=i$.

By means of the Lookup query, the dictionary is able to answer membership queries in an exact way (rather than approximate) and to associate satellite information to $k$-mers (such as abundances). Thanks to the Access query, the original set $\mathcal{S}$ can be reconstructed, meaning that the dictionary is a self-index for $\mathcal{S}$.

In sequence analysis tasks, it is very often the case that we are given a pattern $P$ of length $|P| \geq k$ and we are interested in answering membership to $\mathcal{S}$ for all the $k$-mers read consecutively from $P$, that is, for $P[i, i+k), i=0, \ldots,|P|-k$. For example, we may decide that the whole pattern $P$ is present in a genome if the number of $k$-mers of $P$ that belong to $\mathcal{S}$ is at least $\theta|P|$, for a prescribed coverage threshold $\theta>0$, such as $\theta=0.8$ [Bingmann et al., 2019]. In other words, Lookup queries are often issued for consecutive $k$-mers (one being the previous shifted to the right by one symbol) [Robidou and Peterlongo, 2021, Bingmann et al., 2019]. While it is obviously possible to perform $|P|-k+1$ Lookup queries for a pattern of length $|P|$, it also seems profitable to answer "Is $P[i, i+k)$ a member of $\mathcal{S}$ ?" more efficiently knowing that the previous $k$-mer shares $k-1$ symbols with $P[i, i+k)$. We regard this latter scenario as that of streaming queries.

Therefore, our objective is to support Lookup, Access, and streaming membership queries as efficiently as possible in compressed space. (The data structure is static: insertions/deletions of $k$-mers are not supported.)

As a first introductory remark we shall mention that the algorithmic literature about the so-called compressed string dictionary problem is rich of solutions, e.g., based on Front-Coding, tries, hashing, or combinations of such techniques (see the survey by Martínez-Prieto et al. [2016]) However, these solutions are unlikely to be competitive for the specialized version of the problem we tackle here because they are relevant for "generic" strings that usually: (1) have variable length; (2) are drawn from larger alphabets (e.g., ASCII); (3) do not exhibit particular properties that can aid compression. Instead, $k$-mers are fixed-length strings; their alphabet of representation is very small (just 2 bits per symbol are sufficient); and since $k$-mers are extracted consecutively from DNA, two consecutive strings overlap by $k-1$ symbols that are redundant and should not be represented twice in the dictionary. This motivates the study of specialized solutions for $k$-mers.

These properties are elegantly captured by the de Bruijn graph representation of $\mathcal{S}$ - a graph whose nodes are the $k$-mers in $\mathcal{S}$ and the edges model the string overlaps between the $k$-mers. Using this formalism, it is possible to reduce the redundancy of the symbols in $\mathcal{S}$ by considering paths in the graph and their corresponding strings, such as the maximal unitigs (or the maximal stitched unitigs [Rahman and Medvedev, 2020]). We will better formalize these notions in Section 2. 
For the scope of this work, it is sufficient to point out that: (1) many algorithms have been proposed to build de Bruijn graphs efficiently [Chikhi et al., 2016, Khan and Patro, 2021, Khan et al., 2021] from which these paths can be extracted for indexing purposes; (2) not surprisingly, essentially all state-of-the-art dictionaries for $k$-mers - that we briefly review in Section 3 - are based on the principle of indexing such collections of paths [Almodaresi et al., 2018, Chikhi et al., 2014, Marchet et al., 2021, Rahman and Medvedev, 2020]. We also follow this direction.

However, we note that existing dictionary data structures either represent such paths with an FM-index [Ferragina and Manzini, 2000] (or one of its many variants), hence retain highly compressed space but very slow query time in practice or, vice versa, resort on hashing for fas evaluation but take much more space [Almodaresi et al., 2018, Marchet et al., 2021]. It is, therefore, desirable to have a good balance between these two extremes.

For this reason, we show that we can still enjoy the query efficiency of hashing while taking small space - significantly less space than prior schemes based on (minimal and perfect) hashing. More specifically, we show how two statistical properties of $k$-mer minimizers - precisely, those of being sparse and skewly distributed in DNA sequences - can be better exploited to derive an efficient dictionary data structure based on minimal perfect hashing and compact encodings (Section 4). We evaluate the proposed dictionary data structure over sets of billions of $k$-mers, under different query distributions and modalities, and exhibit a substantial performance improvement compared to prior solutions for the problem (Section 5). Our C++ implementation of the dictionary is available at https://github.com/jermp/sshash.

\section{Preliminaries}

In this section we give some preliminary remarks to better support the exposition in Section 3 and 4. Let $\mathcal{S}$ be the collection of the $n$ distinc $k$-mers extracted from a given, large, string (or set of strings). This string can be, for example, the genome of an organism. Throughout the paper, we consider as identical two $k$-mers that are the reverse complement of each other.

de Bruijn Graphs and Paths. A (node-centric) de Bruijn graph (dBG) for $\mathcal{S}$ is a directed graph $G(\mathcal{S})$ where the set of nodes is $\mathcal{S}$ and a direct edge from node $u$ to $v$ exists if and only if the last $k-1$ symbols of $u$ are equal to the first $k-1$ symbols of $v$, i.e., $u[1, k-1]=v[0, k-2]$. With a little abuse of notation, we refer to a path in $G(\mathcal{S})$ or to the string spelled out by the path (the string obtained by "glueing" all the nodes' $k$-mers in order) interchangeably.

A unary path (or unipath) of length $\ell$ in a $\mathrm{dBG}$ is a path made up $\ell$ nodes that all have in-degree and out-degree equal to 1 except, possibly, the in-degree of the first node and the out-degree of the last node of the path. A maximal unary path is a unary path that cannot be extended without violating the property of being a unary path. Given a maximal unary path of length $\ell$, the corresponding maximal unitig is the string of length $\ell+k-1$ spelled out by the path.

We say that $G_{c}(\mathcal{S})$ is the compacted $\mathrm{dBG}$ of $G(\mathcal{S})$ if the nodes of $G_{c}(\mathcal{S})$ are the maximal unitigs of $G(\mathcal{S})$. A stitched unitig is obtained by following a path in $G_{c}(\mathcal{S})$. A disjoint-node path cover for $G_{c}(\mathcal{S})$ is a set of paths where each node belongs to exactly one path. Efficient heuristic algorithms have been proposed to approximate a disjoint-node path cover for $G_{c}(\mathcal{S})$ that is of minimum size (in the number of paths); see, for example, that by Rahman and Medvedev [2020]. We denote such a cover with $\mathcal{S}^{\prime}$. The strings in $\mathcal{S}^{\prime}$, therefore, form the natural basis for a space-efficient dictionary because:
- by trying to minimize the number of paths in the cover, the number of symbols in $\mathcal{S}^{\prime}$ is less than the number of symbols in $\mathcal{S}$ and,

- by being a disjoint-node path cover we are guaranteed that there are no duplicate $k$-mers in $\mathcal{S}^{\prime}$.

In conclusion, we assume from now on that a path cover $\mathcal{S}^{\prime}$ has been computed for $G_{c}(S)$ as the input for our problem.

Minimizers and Super- $k$-mers. Given a $k$-mer $g$, an integer $m \leq k$, and a total order relation $R$ on all $k$-length strings, the smallest $m$-mer of $g$ according to $R$ is called the minimizer of $g$. $R$ could be, for example, the simple lexicographic order. Instead, here we use the random order given by a hash function $h$, chosen from a universal family ${ }^{1}$. Therefore, simply put, the minimizer of $g$ is the $m$-mer of $g$ that minimizes the value of $h$.

Minimizers are very popular in sequence analysis, such as for seedand-extend algorithms, because of the following empirical property: consecutive $k$-mers tend to have the same minimizer [Schleimer et al., 2003, Roberts et al., 2004]. This means that there are far less distinct minimizers than $k$-mers; approximately $(k-m+2) / 2$ times less minimizers than $k$-mers (independently of the sequence length), if $m$ is not very smal compared to $k$ [Schleimer et al., 2003, Zheng et al., 2020]. For example, if $k=31$ and $m=20$, we should expect to see roughly $(31-20+2) / 2=6.5 \times$ less minimizers than $k$-mers. Another way of picturing this fact is to say that we approximately sample a minimizer every $(k-m+2) / 2$ symbols

We call a super- $k$-mer a maximal sequence of consecutive $k$-mers having the same minimizer [Li et al., 2013].

Minimal Perfect Hashing. Given a set $\mathcal{X}$ of $n$ distinct keys, a function $f$ that maps bijectively the keys into the integer range $\{0, \ldots, n-1\}$ is called a minimal perfect hash function (MPHF) for the set $X$. The function is allowed to return an arbitrary value in $[0, n)$ for any key that does not belong to $X$, hence it can be realized in small space, in practice $2-3$ bits/key (albeit $\log _{2} e \approx 1.44$ bits/key are sufficient in theory [Mehlhorn, 1982]). Many efficient algorithms have been proposed to build MPHFs from static sets that scale well to large values of $n$ and retain practicallyconstant evaluation time. In this paper, we use PTHash [Pibiri and Trani, 2021a,b] for its very fast evaluation time, usually $2-4 \times$ better than other techniques, and good space effectiveness.

\section{Related Work}

As already anticipated in Section 1, most existing solutions for exact membership queries are based on indexing the unitigs of the de Bruijn graph. These approaches have also been summarized in the recent survey by Chikhi et al. [2021, Section 4.2], hence we give a rather cursory overview here.

The maximal unitigs can be represented using an FM-index [Ferragina and Manzini, 2000] for very compact space [Chikhi et al., 2014, Rahman and Medvedev, 2020]. The practical efficiency of the FM-index mainly depends on how many samples of the suffix-array are kept in the index.

Other approaches resort on hashing for fast lookup queries. For example, Bifrost [Holley and Melsted, 2020] uses a hash table of minimizers whose values are the locations of the minimizers in the unitigs. The index was designed to be dynamic, hence allowing insertion/removal of $k$-mers and consequent re-computation of the unitigs. The dynamic nature of Bifrost makes it consume higher space compared to static approaches using compressed hash representations and succinct data structures, like Pufferfish [Almodaresi et al., 2018] and Blight [Marchet et al., 2021]. Hence, it is regarded as out of scope for this work.

${ }^{1}$ In our implementation, we use an instance of MurmurHash [Appleby, 2016] with a given seed. 
Pufferfish [Almodaresi et al., 2018] associates to each $k$-mer its location in the unitigs using a MPHF and a vector of absolute positions. The authors also proposed a sparse version of the index where the vector of positions is sampled to improve space usage at the expense of query time. Blight [Marchet et al., 2021] is also an associative dictionary based on minimal perfect hashing. It partitions the $k$-mers by minimizers and builds a separate MPHF on each partition to improve space usage compared to Pufferfish. To further reduce space, a $k$-mer is associated to the segment of $2^{b}$ super- $k$-mers where it belongs to, for a given $b \geq 0$. This reduces the space of the dictionary by $b$ bits per $k$-mer but a lookup needs to scan (at most) $2^{b}$ super- $k$-mers

Very importantly, Pufferfish and Blight are optimized for streaming membership queries.

\section{Sparse and Skew Hashing}

In this section we describe our main contribution: an exact, associative, and compressed dictionary data structure for $k$-mers, supporting fas Lookup, Access, and streaming queries. From a high-level point of view, the dictionary is obtained via a careful combination of minimal perfect hashing and compact encodings: in particular, we show how two important properties of minimizers - those of being sparse (Section 4.1) and skewly distributed (Section 4.2) in DNA strings - can be exploited to achieve an efficient dictionary. Our focus is on supporting fast queries in compressed space, thus we aim at a good trade-off between dictionary space and query efficiency.

Recall from Section 2 that the dictionary is built from a collection of paths covering a de Bruijn graph (for example, the maximal stitched unitigs), that is: a collection of strings, each of length at least $k$ symbols, with no duplicate $k$-mers. For ease of notation, we indicate with $p$ the number of paths in the collection and with $N$ their cumulative length. The total number of (distinct) $k$-mers is, therefore, $n=N-p(k-1)$.

\subsection{Sparse Hashing}

The starting point for our development is based on the well-known empirical property of minimizers in that consecutive $k$-mers are likely to have the same minimizer. Thus, instead of working with individual $k$-mers, we focus on maximal sequences of $k$-mers having the same minimizer - the so-called super- $k$-mers (see Section 2). Super- $k$-mers are useful because of the following two reasons.

- As super- $k$-mers are likely to span several consecutive $k$-mers, we expect to see far fewer super- $k$-mers than $k$-mers, roughly $(k-m+2) / 2$ times less for a, sufficiently-large, chosen minimizer length $m$ Informally, this property allows a space usage proportional to the number of super- $k$-mers, thus sparsifying the dictionary.

- A super- $k$-mer of length $s$ is a space-efficient representation for its constituent $s-k+1 k$-mers since it takes $2 s /(s-k+1)$ bits $/ k$-mer instead of the trivial cost of $2 k$ bits $/ k$-mer.

Therefore, our refined ambition is to index the super- $k$-mers of the input. Although this can simply be achieved by inserting the super- $k$-mers into a (minimal and perfect) hash table-i.e., by laying out the super- $k$-mers in the order given by a hash function - we claim that his approach is very wasteful in terms of space. Note that each super- $k$-mer has a fixed cost of $2(k-1)$ bits for representing the "tail" of its string (its last $k-1$ symbols). If the super- $k$-mer has length $s$, this fixed cost is only well amortized (say, negligibly small) when $s$ is much larger than $k-1$. In other words, when the super- $k$-mer contains many more $k$-mers than $k-1$. While possible in same extreme cases (e.g., the same minimizer repeats in sequence), it is not usually so for the values of $k$ and $m$ used in concrete applications; actually, a super- $k$-mer is more likely to contain $k-m+1 k$-mers or less.

If $z$ indicates the number of super- $k$-mers in the input, then the space of this simple solution would be, at least, $2+2 z(k-1) / n$ bits/ $k$-mer (extra space is then needed to accelerate the queries). For example, consider the whole human genome with $k=31$ and $m=20$. There are more than $z=396 \times 10^{6}$ super- $k$-mer for, roughly, $n=2.5 \times 10^{9}$ distinct $k$-mers Therefore, partitioning the strings according to super- $k$-mers would cost at least $11.50 \mathrm{bits} / k$-mer. As we will better see in Section 5, our dictionary can be tuned to take $8.28 \mathrm{bits} / k$-mer overall in this case (or less).

Thus, it is of utmost importance to not break the strings according to super- $k$-mers if space-efficiency is a concern. Instead, we identify a super$k$-mer in the strings, whose total length is $N$, with an absolute offset of $\left\lceil\log _{2}(N)\right\rceil$ bits. To be precise, an offset is the position in $[0, N)$ of the first base of a super- $k$-mer. Since $k$ should be chosen large enough to allow good $k$-mer specificity, $2(k-1)$ will be much larger than $\left\lceil\log _{2}(N)\right\rceil$ in practice, even for the largest genomes. For example, we use $k=31$ in our experiments, as done in many other works [Almodaresi et al., 2018, Marchet et al., 2021, Rahman and Medvedev, 2020, Bingmann et al., 2019], whereas $\left\lceil\log _{2}(N)\right\rceil$ is around $30-34$ for collections with billions of $k$ mers (see also Table 2 at pag. 6). The use of absolute offsets can almost halve the space overhead for the indexing of super- $k$-mers in such cases. The space saving is even larger for larger $k$.

Dictionary Layout and Compression. Based on the above discussion, we now detail the different components of our dictionary data structure.

1. Strings. The $p$ strings in the input collections are written one after the other in a vector of $2 N$ bits ( 2 bits per input base). We also materialize in a sorted integer sequence of length $p$ the endpoints of the strings to avoid detection of alien $k$-mers. This sequence, Endpoints, is compressed with Elias-Fano [Fano, 1971, Elias, 1974] and takes $p\left\lceil\log _{2}(N / p)\right\rceil+2 p+o(p)$ bits.

2. Minimizers. Let $\mathcal{M}$ be the set of all distinct minimizers seen in the input, with $M=|\mathcal{M}|$, and $z$ the number of super- $k$-mers. Clearly, we have $z \geq M$ because a minimizer can appear more than once in the input. Given a minimizer $r$, let us call the bucket of the minimizer $r, B_{r}$, the set of all the super- $k$-mers that have minimizer $r$. We build a minimal perfect hash function $f$ for $\mathcal{M}$. The MPHF provides us an addressable space of size $M$ : the value $f(r) \in[0, M)$ is the "bucket identifier" of $r, r \in \mathcal{M}$. We materialize an array Sizes $[0, M+1)$, where Sizes $[f(r)+1]=\left|B_{r}\right|$ is the size of the bucket of $r$, and Sizes $[0]=0$. We then take the prefix-sums of Sizes, i.e., we replace Sizes $[i]$ with Sizes $[i]+\operatorname{Sizes}[i-1]$ for all $i>0$. Therefore, for a given minimizer $r$, now Sizes $[f(r)]$ indicates that there are Sizes $[f(r)]$ buckets before $B_{r}$ in the order given by $f$.

The function $f$ costs roughly 3 bits per minimizer; the Sizes array is compressed with Elias-Fano too and takes $M\left\lceil\log _{2}(z / M)\right\rceil+2 M+$ $o(M)$ bits.

3. Offsets. The absolute offsets of the super- $k$-mers into the strings are stored in an array, Offsets $[0, z)$, in the order given by $f$. For a minimizer $r$ such that Sizes $[f(r)]=$ begin, its $\left|B_{r}\right|$ offsets are written consecutively (and in sorted order) in Offsets[begin, begin $\left.+\left|B_{r}\right|\right)$. Note that, by construction, Sizes $[f(r)+1]-\operatorname{Sizes}[f(r)]=\left|B_{r}\right|>0$. The space for the array is $z\left\lceil\log _{2}(N)\right\rceil$ bits.

Summing up the different costs, the dictionary space is then $2 N+5 M+$ $z\left\lceil\log _{2}(N)\right\rceil+M\left\lceil\log _{2}(z / M)\right\rceil+p\left\lceil\log _{2}(N / p)\right\rceil+2 p+o(p)+o(M)$ bits. Basically all the space is due to the first four terms, $2 N+5 M+$ $z\left\lceil\log _{2}(N)\right\rceil+M\left\lceil\log _{2}(z / M)\right\rceil$, since $p$ is at least $(k-1)$ times smaller than $N$ (in practice, much smaller) and lower-order terms are very small. 
Queries and Double Strandedness. Now we describe how the Lookup and Access queries are supported. We first consider Lookup. Given a $k$-mer $g$, we compute its minimizer $r$ and its bucket identifier as $f(r)$. Then, we locate the super- $k$-mers in its bucket $B_{r}$ by retrieving the corresponding offsets from Offsets[begin, end), where begin $=\operatorname{Sizes}[f(r)]$ and end $=$ $\operatorname{Sizes}[f(r)+1]$. For every offset $t$ in Offsets[begin, end $)$, we scan the super- $k$-mer starting from Strings $[t]$ comparing its $k$-mers to the query $g$. If $g$ is not found, we just return -1 . Instead, if $g$ is found in position $w$ in the super- $k$-mer, we return the "identifier" $i$ of $g$ as $i=t-j(k-1)+w$ where $j<p$ is the number of strings before the one containing the offset $t$ (this quantity is computed from the Endpoints array).

Note that, by construction, we cannot compute the length of a super- $k$ mer from the Offsets array (at least, not efficiently). That is, the offset $t$ only indicates that a super- $k$-mer begins at Strings $[t]$. So we start comparing the $k$-mer at Strings $[t]$ but we do not know exactly after how many symbols we can stop the search. We could re-compute minimizers from the $k$-mers read during the scan of Strings to derive this information but we want to avoid doing so. In fact, we know that a super- $k$-mer $g^{\prime}$ cannot contain more than $k-m+1 k$-mers unless the same minimizer appears again somewhere in $g^{\prime}[0,2 k-m)$. Albeit rare, the latter case is possible and it is handled as follows. If a super- $k$-mer is longer than $2 k-m$ symbols, it is split into blocks of $2 k-m$ symbols (except possibly the last one) and we just pretend that each block is a distinct super- $k$-mer. Thus, at query time it is safe to consider a window of $\min \left(2 k-m, t_{e n d}-t\right)$ symbols, where $t_{\text {end }} \geq t+k$ is the successor of the offset $t$ in Endpoints (i.e., $t_{\text {end }}$ is the smallest value in Endpoints that is $\geq t$ ). Capping the window to $t_{e n d}-t$ symbols if $t_{\text {end }}-t<2 k-m$ is necessary to not falsely consider alien $k$-mers that are formed at the boundary between two consecutive strings in the compact vector Strings.

Summing up, we map a $k$-mer $g$ to its bucket of super- $k$-mers in constant time by issuing, approximately, 4 cache misses: 1 to compute $f$ since we use the PTHash data structure, 3 for the accesses to Sizes and Offsets. Then we perform at most end - begin cache misses for the accesses to Strings, plus an extra access to the Endpoints array. Since the scan of consecutive symbols is very cache-friendly, a Lookup query essentially pays the cost of the indicated cache misses.

A detail of crucial importance to discuss is double strandedness. A $k$-mer and its reverse complement are considered as identical. This means that if a $k$-mer $g$ is not found by the Lookup algorithm, there can stil be the possibility for its reverse complement $\hat{g}$ to be found in Strings. Therefore, the actual Lookup routine will first search for $g$ and, only if not found, will also search for $\hat{g}$. This effectively doubles the query time for Lookup in the worst case. To guarantee that a Lookup will always inspect one single bucket, we use a different minimizer computation (during both query and dictionary construction): we select as minimizer the minimum between the minimizer of $g$ and that of $\hat{g}$. In this way, it is guaranteed that two $k$-mers being the reverse complements of each other always belong to the same bucket. This different minimizer selection actually changes the parsing of super- $k$-mers from the input during the construction of the dictionary. We refer to this parsing modality as canonical henceforth, in contrast to the regular modality we assumed so far. When this modality is chosen, we expect to see an increase in the number of distinct minimizers used (on average, the minimizers of $g$ and $\hat{g}$ have equal probability of being the minimum one) for a higher space usage, but faster query times We will explore the space/time trade-off between the regular and canonical modalities in Section 5.1.

Lastly, the Access query extracts the $k$-mer $g$ given its identifier $i$. All that is needed is a binary search for $i$ in Endpoints to derive the offset $t$ corresponding to $i$. Once the offset $t$ is computed, we return $g=$ Strings $[t, t+k)$.
It is therefore obvious that the minimizer length $m$ controls a space/time trade-off. Small values of $m$ create fewer and longer super- $k$-mers, thus lowering the space for the smaller values of $z$ and $M$. On the other hand, $m$ should not be chosen too small to avoid the scan of many super- $k$-mers at query time. We will experimentally show the trade-off in Section 5.1. Next, we take a deeper look at lookup time.

\subsection{Skew Hashing}

The efficiency of the Lookup query depends on the number of super- $k$ mers in the bucket of a minimizer, which we refer to as the "size" of the bucket. Since a minimizer can appear multiple times in the input strings, nothing prevents its bucket size to grow unbounded. For example, on the human genome, the largest bucket size can be as large as $3.6 \times 10^{4}$ for $m=20$ (or even larger for smaller values of $m$ ), meaning that a query inspecting such a bucket would be very slow in practice. To avoid the burden of these heavy buckets, i.e., to guarantee that a Lookup inspects a constant number of buckets in the worst case, we exploit another importan property of minimizers: the distribution of the bucket size is (very) skewed for sufficiently large $m$. That is, most minimizers appear just once and relatively few of them repeat many times. Moreover for a fixed value of $n$, the distribution of bucket size is essentially independent from a specific input, rather it depends on $k$ and $m$. Table 1 shows such distribution fo some useful values of $n$ and $k=31$, that is, the percentage of super- $k$ mers falling into buckets of size $\left|B_{r}\right|=1,2,3, \ldots$ (we show the first 5 sizes). The $n$ values in the table mimic the ones used in our experiment in Section 5. As apparent, for each $n$, there is a value of $m$ for which the distribution is very skewed, e.g., most buckets (> 90\%) contain just 1 super- $k$-mer. We want to take advantage of this distribution.

We fix two quantities $\ell$ and $L$, with $L>\ell \geq 0$. By virtue of the skew distribution, we have that the number of buckets whose size is larger than $2^{\ell}$ is small, as well as the number of $k$-mers belonging to such buckets. This allows us to, again, build a minimal perfect hash function to speed up query processing but only for a small fraction of the total $k$-mers. For ease of exposition, in the following we assume that $2^{L} \leq \max$, where $\max$ is the largest bucket size (the corner case for $2^{\ell} \leq \max <2^{L}$ is then easy to obtain).

For $\ell \leq i \leq L$, let $\mathcal{S}_{i}$ be the set of all the $k$-mers belonging to buckets of size

$$
\left\{\begin{array}{l}
2^{i}<s \leq 2^{i+1} \quad \ell \leq i<L \\
2^{L}<s \leq \max \quad i=L
\end{array}\right.
$$

We build a MPHF $f_{i}$ for each set $\mathcal{S}_{i}$. Now, given a $k$-mer $g \in \mathcal{S}_{i}$, we know that it belongs to a bucket containing at most $2^{i+1}$ super- $k$-mers. Therefore, we can store the identifier of the super- $k$-mer containing $g$ in a vector, say $P_{i}$, of $\left|\mathcal{S}_{i}\right|$ integers at position $f_{i}(g)$. Importantly, each integer in $P_{i}$ requires just $i+1$ bits $\left(P_{L}\right.$ is formed by $\left\lceil\log _{2} \max \right\rceil$-bit integers $)$. We point out that, again thanks to the skew distribution, it is very likely to have $\left|\mathcal{S}_{\ell}\right| \geq\left|\mathcal{S}_{\ell+1}\right| \geq \cdots \geq\left|\mathcal{S}_{L-1}\right|$. Therefore, for a proper choice of $\ell$ and $L$, we expect this additional skew index component of the dictionary to take little space, while granting very fast searches.

To make a concrete example, let us consider the human genome and the skew index with $\ell=6$ and $L=12$. So we form $L-\ell+1=12-6+1=7$ partitions; each partition is made up of a MPHF and a compact vector. Each MPHF $f_{i}$ can be tuned to take $2.5-3.0$ bits/ $k$-mer, whereas we spend $i+1 \mathrm{bits} / k$-mer for $P_{i}, i=6, \ldots, 11$. As already mentioned, $\max =3.6 \times 10^{4}$ for $m=20$, thus we spend $\left\lceil\log _{2} \max \right\rceil=16 \mathrm{bits} / k$-mer in the last partition. The crucial point is that we have $0.016 \%$ of buckets that comprise more than $2^{6}$ super- $k$-mers, for just $1.86 \%$ of the total $k$ mers. For this reason, the skew index costs $0.64 \%$ of the total dictionary space (i.e., $0.21 \mathrm{bits} / k$-mer overall). 
Table 1. Bucket size distribution (\%) for $k=31$ and some useful values of $n$, by varying $m$.

\begin{tabular}{crrrrrrrrrrr}
\hline size $/ m$ & 11 & 12 & 13 & 14 & 15 & 16 & 17 & 18 & 19 & 20 & 21 \\
\hline 1 & 16.1 & 24.0 & 35.3 & 51.3 & 71.6 & 85.9 & 92.8 & 95.6 & 96.8 & 97.4 & 97.7 \\
2 & 8.7 & 12.6 & 15.7 & 19.7 & 17.0 & 10.0 & 5.2 & 2.9 & 2.0 & 1.6 & 1.4 \\
3 & 6.1 & 8.3 & 9.4 & 10.4 & 5.7 & 2.2 & 1.0 & 0.6 & 0.5 & 0.4 & 0.4 \\
4 & 4.6 & 6.0 & 6.6 & 6.1 & 2.3 & 0.8 & 0.4 & 0.3 & 0.2 & 0.2 & 0.2 \\
5 & 3.8 & 4.6 & 5.1 & 3.7 & 1.1 & 0.4 & 0.2 & 0.1 & 0.1 & 0.1 & 0.1 \\
\hline
\end{tabular}

(a) $n=0.5 \times 10^{9}$

\begin{tabular}{crrrrrrrrrrr}
\hline size $/ m$ & 11 & 12 & 13 & 14 & 15 & 16 & 17 & 18 & 19 & 20 & 21 \\
\hline 1 & 13.7 & 19.8 & 29.7 & 42.4 & 61.5 & 79.5 & 89.8 & 94.4 & 96.3 & 97.1 & 97.5 \\
2 & 7.5 & 10.6 & 14.4 & 17.7 & 19.4 & 13.6 & 7.3 & 3.9 & 2.4 & 1.7 & 1.4 \\
3 & 5.2 & 7.3 & 8.8 & 10.4 & 8.4 & 3.7 & 1.4 & 0.8 & 0.5 & 0.4 & 0.4 \\
4 & 4.0 & 5.5 & 6.0 & 7.0 & 4.1 & 1.3 & 0.5 & 0.3 & 0.2 & 0.2 & 0.2 \\
5 & 3.2 & 4.4 & 4.5 & 5.0 & 2.2 & 0.6 & 0.3 & 0.2 & 0.1 & 0.1 & 0.1 \\
\hline
\end{tabular}

(b) $n=1.0 \times 10^{9}$

\begin{tabular}{crrrrrrrrrrr}
\hline size $/ m$ & 11 & 12 & 13 & 14 & 15 & 16 & 17 & 18 & 19 & 20 & 21 \\
\hline 1 & 11.2 & 15.6 & 23.0 & 33.9 & 48.2 & 67.8 & 83.2 & 91.3 & 94.9 & 96.4 & 97.1 \\
2 & 6.1 & 8.5 & 12.1 & 15.3 & 18.9 & 18.0 & 11.4 & 6.2 & 3.5 & 2.3 & 1.8 \\
3 & 4.3 & 5.9 & 8.1 & 9.1 & 10.4 & 6.7 & 2.8 & 1.2 & 0.7 & 0.5 & 0.4 \\
4 & 3.3 & 4.5 & 5.9 & 6.3 & 6.5 & 3.0 & 1.0 & 0.4 & 0.3 & 0.2 & 0.2 \\
5 & 2.7 & 3.7 & 4.6 & 4.8 & 4.2 & 1.5 & 0.5 & 0.2 & 0.2 & 0.1 & 0.1 \\
\hline
\end{tabular}

(c) $n=2.5 \times 10^{9}$

\begin{tabular}{crrrrrrrrrrr}
\hline size $/ m$ & 11 & 12 & 13 & 14 & 15 & 16 & 17 & 18 & 19 & 20 & 21 \\
\hline 1 & 8.7 & 11.2 & 15.1 & 23.6 & 41.4 & 62.9 & 78.2 & 86.9 & 91.6 & 94.1 & 95.4 \\
2 & 4.8 & 6.1 & 8.4 & 13.5 & 19.9 & 19.1 & 13.7 & 9.2 & 6.4 & 4.7 & 3.8 \\
3 & 3.3 & 4.2 & 6.0 & 9.6 & 11.5 & 7.7 & 4.1 & 2.2 & 1.3 & 0.8 & 0.6 \\
4 & 2.5 & 3.3 & 4.7 & 7.3 & 7.2 & 3.7 & 1.6 & 0.8 & 0.4 & 0.2 & 0.1 \\
5 & 2.1 & 2.7 & 3.9 & 5.9 & 4.8 & 2.0 & 0.8 & 0.4 & 0.2 & 0.1 & 0.1 \\
\hline
\end{tabular}

(d) $n=5.0 \times 10^{9}$

Using the skew index to accelerate $\operatorname{Lookup}(g)$ is simple. As for regular Lookup, we compute the minimizer $r$ of $g$ and the quantities begin $=$ Sizes $[f(r)]$ and end $=$ Sizes $[f(r)+1]$. Therefore we know that the bucket of $r$ has size end - begin $\leq$ max. Let $b=\left\lceil\log _{2}(\right.$ end - begin $\left.)\right\rceil$. If $b \leq \ell$, then the bucket is "small" and we proceed as already explained in Section 4.1 Otherwise we know that $g$, if present in the dictionary, belongs to some partition $i$ of the skew index that as per our description above, has MPHF $f_{i}$ and compact vector $P_{i}(i=b$ if $b \leq L$ or $i=L$ otherwise). Thus, we compute the super- $k$-mer identifier as $q=P_{i}\left[f_{i}(g)\right]$ and finally search for $g$ in the super- $k$-mer whose offset is Offsets [begin $+q]$. (Note that if $q \geq e n d$, then $g$ cannot belong to the dictionary.) In conclusion, although the skew index performs 2 additional accesses per Lookup, one for $f_{i}$ and one for $P_{i}$, it limits the number of accesses made to Strings to $2^{\ell}$.

(To handle reverse complements, we may have to repeat the process also for the reverse complement of $g$, as pointed out previously.)

\subsection{Streaming Queries}

The Lookup algorithm we have described in the previous section is contextless, i.e., it does not take advantage of the specific, consecutive, query order issued by sequence analysis tasks. As already mentioned in Section 1 , given a string $P$ of length $|P| \geq k$, we are interested in determining the result of Lookup for all the $k$-mers read consecutively from $P$. We would like to do it faster than just performing $|P|-k+1$ independent lookups. Therefore, in this section we describe some important optimizations for streaming lookup queries that work well with the proposed dictionary data structure. The general idea is to cache some extra information about the result for the $k$-mer $g=P[i, i+k)$ to speed up the computation for the next $k$-mer in $P$, say $g_{n x}=P[i+1, i+k+1)$

The algorithm keeps track of the minimizer $r$ of $g$ and the position $j$ at which the last match was found in Strings, i.e., if $g$ belongs to the dictionary, then it is located at Strings $[j, j+k)$ for some $j$. These two variables make up a state information that is updated during the execution of the algorithm. Given that consecutive $k$-mers are likely to share the same minimizers, we compare $r$ to the minimizer of $g_{n x}$, say $r_{n x}$.

- If $r_{n x}=r$, then we know that $g_{n x}$ belongs to the same bucket $B_{r}$ of $g$, thus we avoid recomputing $f$ and spare the accesses to both Sizes and Offsets. Also, if $g$ was actually found in the dictionary (therefore, starting at Strings $[j]$ ) good chances are that $g_{n x}$ is found at $\operatorname{Strings}[j+$

1]. If so, we refer to the latter matching case as an extension. Intuitively, if the algorithm "extends" frequently, i.e., most matches in $P$ are determined by just looking at consecutive $k$-mers in Strings, then fast evaluation is retained. If the algorithm does not extend from $g$ to $g_{n x}$, i.e., $g_{n x}$ is not found at Strings $[j+1]$, then we scan the bucket $B_{r}$. Therefore, if present in the dictionary, $g_{n x}$ will be found at some other position $j_{n x}$. So we update the state by setting $j=j_{n x}$.

- If $r_{n x} \neq r$, then we proceed as for a regular Lookup query, locating the new bucket $B_{r_{n x}}$ and searching for $g_{n x}$. We then set $r=r_{n x}$.

Of course it can happen that the minimizer $r$ does not belong to the set of minimizers indexed by the dictionary. Recall from Section 4.1 that we build the MPHF $f$ for the set $\mathcal{M}$ of all the distinct minimizers in the input. In this case, we are sure that any $k$-mer $g$ whose minimizer $r \notin \mathcal{M}$ is not to be found in the dictionary. By definition, however, we are not able to detect if $r \notin \mathcal{M}$ using the MPHF $f$. That is, $f$ will still locate a bucket and all the $k$-mers in the bucket will have (the same) minimizer different from $r$. Therefore, when searching for $g$, we first compare $r$ with the minimizer of the first $k$-mer read in the bucket: if they are different, we know that $r \notin \mathcal{M}$ and $g$ does not belong to the dictionary. In the case when $r \notin \mathcal{M}$, the algorithm still caches the last seen minimizer because if $r_{n x}=r$ then also $r_{n x} \notin \mathcal{M}$ and $g_{n x}$ cannot belong to the dictionary.

In conclusion, as long as the minimizer is the same, either the algorithm works locally in the same bucket, or safely skips the computation.

Another convenient information to cache in the state of the algorithm is the orientation of the last match, that is, whether the last queried $k$-mer $g$ was found in the dictionary as $g$ or as its reverse complement $\hat{g}$. In fact, if $g$ was found as $g$ then also $g_{n x}$ is likely to be found as $g_{n x}$ and extension should be tried in forward direction (say, from lower to higher offsets in Strings). But if $g$ was found as $\hat{g}$, then is more efficient to try to extend the matching in backward direction, hence effectively iterating backwards in Strings. In fact, suppose that the whole string $P$ (for ease of exposition) is present in Strings but in its reverse complement form. Then the first $k$-mer $g$ of $P$ will be found as $\hat{g}$ in last position in the located "region" of Strings, say at some position $j$. Any other attempt to extend the matching in forward direction (from $j$ to $j+1$ ) will then fail and any subsequent $g_{n x}$ will be searched for by re-scanning the bucket again. That is, we end up in scanning the bucket for $c=|P|-k+1$ times, for at least $O\left(c^{2}\right) k$-mer comparisons. To prevent this quadratic behavior in case of reverse complemented patterns, we try to directly extend the matching for $g_{n x}$ moving from $j$ to $j-1$.

Lastly, it is also convenient to optimize the computation of the minimizers when working on streams. Instead of paying $\Theta(k-m+1)$ time and $O(1)$ space to compute each minimizer, it is possible to spend $O(1)$ amortized per minimizer and a global working space of $O(k-m+1)$ using an adaptation of the best algorithm to solve the sliding window minimum problem. 
Table 2. Some basic statistics for the datasets used in the experiments, for $k=31$, such as number of: $k$-mers $(n)$, paths $(p)$, and bases $(N)$.

\begin{tabular}{lrrrc}
\hline Dataset & $n$ & $p$ & $N$ & $\left\lceil\log _{2}(N)\right\rceil$ \\
\hline Cod & $502,465,200$ & $2,406,681$ & $574,665,630$ & 30 \\
Kestrel & $1,150,399,205$ & 682,344 & $1,170,869,525$ & 31 \\
Human & $2,505,445,761$ & $13,014,641$ & $2,895,884,991$ & 32 \\
Bacterial & $5,350,807,438$ & $26,449,008$ & $6,144,277,678$ & 33 \\
\hline
\end{tabular}

\section{Experiments}

In this section we benchmark the proposed dictionary data structure which we refer to as SSHash in the following - and compare it against the indexes reviewed in Section 3. For all our experiments, we fix $k$ to 31 as used in recent prior work [Almodaresi et al., 2018, Marchet et al., 2021, Rahman and Medvedev, 2020, Bingmann et al., 2019].

Our implementation of SSHash is written in $\mathrm{C}++17$ and available at https://github.com/jermp/sshash. For the experiments we report here, the code was compiled with gcc 11.2.0 under Ubuntu 19.10 (Linux kernel 5.3.0, 64 bits), using the flags -03 and - march=native. We do not explicitly use any SIMD instruction in our codebase.

We use a server machine equipped with an Intel i9-9940X processor (clocked at $3.30 \mathrm{GHz}$ ) and $128 \mathrm{~GB}$ of RAM. Each processor has two private levels of cache memory: $2 \times 32 \mathrm{KiB}$ of $\mathrm{L} 1$ cache $(32 \mathrm{KiB}$ for instructions and $32 \mathrm{KiB}$ for data); $1 \mathrm{MiB}$ for $\mathrm{L} 2$ cache. The third level of cache is shared among all processors and spans $\approx 19 \mathrm{MiB}$. All cache lines are 64-byte long.

The reported timings were collected using a single core of the processor. All dictionaries were fully loaded in internal memory before running the experiments. The SSHash dictionaries were also built entirely in internal memory.

Datasets. We downloaded some DNA collections (in .fasta format) and built the compacted de Bruijn graph using the tool BCALM (v2) [Chikhi et al., 2016], without any $k$-mer filtering, to extract the maximal unitigs. We then run the tool UST [Rahman and Medvedev, 2020] to compute the corresponding path covers. Table 2 reports the basic statistics of the path covers. In particular we used the DNA of the following organisms.

- Cod: the whole genome of the atlantic cod (Gadus Morhua);

- Kestrel: the whole genome of the common kestrel (Falco Tinnunculus);

- Human: the whole GRCh38 human genome (Homo Sapiens);

- Bacterial: a collection of more than 8000 bacterial genomes from Almodaresi et al. [2018].

At the code repository https://github.com/jermp/sshash we provide further instructions on how to download and prepare the datasets for indexing.

\subsection{Tuning}

Before comparing SSHash against other dictionaries, we first benchmark SSHash in isolation to fix a suitable choice for $m$ and quantify the impact of the different parsing modalities (regular vs. canonical) that we introduced in Section 4.1. Following our discussion in Section 4.2, we use $\ell=6$ and $L=12$ for all SSHash dictionaries.

To measure query time, we use $10^{6}$ queries and report the mean between 5 measurements. For positive lookups, i.e., those for $k$-mers present in the dictionary, we sampled uniformly at random $10^{6} k$-mers from each collection and use them as queries. Very importantly, 50\% of them were transformed into their reverse complements to make sure we benchmark the dictionaries in the most general case. For negative lookups, we simply use randomly generated $k$-mer strings. For Access, we generated $10^{6}$ integers uniformly at random in the range $[0, n)$ for each collection and extract the corresponding $k$-mer strings.
Table 3. Space in bits/k-mer (bpk) and average Lookup time (indicated by $\mathrm{Lkp}^{+}$for positive queries; by $\mathrm{Lkp}^{-}$for negative) in ns/k-mer for regular and canonical SSHash dictionaries by varying minimizer length $m$. For each dataset, we indicate promising configurations in bold font.

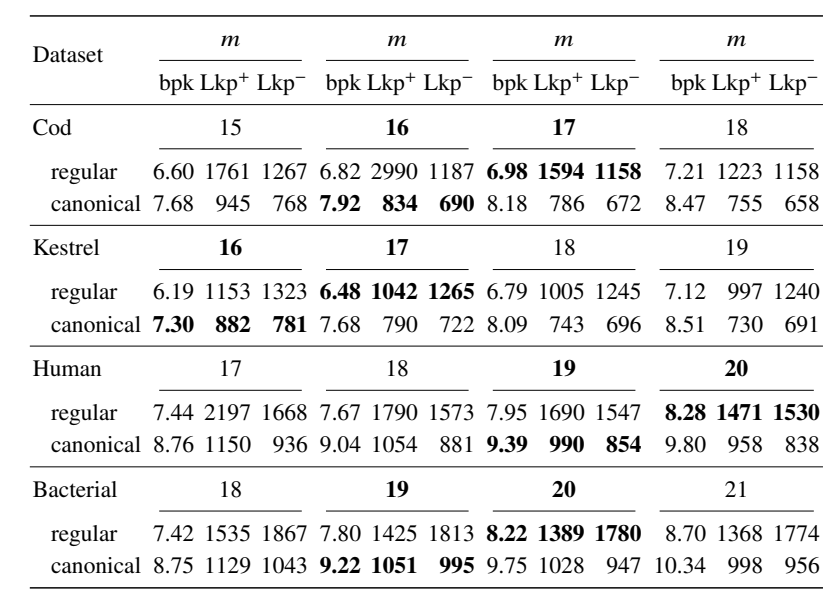

We first recall that the time for Access (and thus, that of iteration) does not depend on $m$ nor $\ell$. The average Access time is, instead, affected by the size of the data structure, i.e., by $n$ and $p$ : Access is on average $2-3 \times$ faster than Lookup since the wanted string is accessed directly, rather than searched for in the dictionary. Iterating thorough all $k$-mers in the dictionary is very fast and even independent from $n$ : on average, it costs $20-22 \mathrm{~ns} / k$-mer. Therefore, for the rest of this section we entirely focus on lookup time.

With the help of Table 1 at pag. 5 we choose some suitable ranges of $m$ for the different dataset sizes. The space/time trade-off by varying $m$ in such ranges, for both regular and canonical parsing modalities, is shown in Table 3. As we discussed in Section 4.1 and apparent from the table, $m$ controls a trade-off between dictionary size and lookup time: the smaller the $m$ value, the more compact the dictionary, but the slower the dictionary as well (and vice versa). While it is difficult to precisely tell by how much the space will grow when moving from $m$ to $m+1$, we see that the space grows by $\approx 0.3-0.4 \mathrm{bits} / k$-mer, for both regular and canonical parsing. The canonical parsing modality costs $\approx 1.0-1.5 \mathrm{bits} / k$-mer more than the regular one for the same value of $m$ because more distinct minimizers are used. However, the canonical version improves lookup time significantly (especially for negative queries), by a factor of $1.4-2.0 \times$ on average, because only one bucket per query is inspected in the worst case rather than two by the regular modality.

Since we seek for a good balance between dictionary space and lookup time, in the light of the results reported in Table 3, we choose:

- for Cod and Kestrel: $m=17$ with regular parsing; $m=16$ with canonical parsing;

- for Human and Bacterial: $m=20$ with regular parsing; $m=19$ with canonical parsing.

The bold font in the table highlights these configurations; in the following, we assume these values of $m$ are used and omit the indication from the tables. In general, we observe that a good value for $m$ satisfies $4^{m}>N$, such as $m=\left\lceil\log _{4}(N)\right\rceil+1$ or $m=\left\lceil\log _{4}(N)\right\rceil+2$. In other words, $m$ should be chosen as to have - at least - as many possible minimizers as the number of bases in the input.

It is also interesting to report how the space is subdivided into the different dictionary components. Fig. 1 shows an example of space breakdown for both regular and canonical dictionaries built from the Human dataset. Very similar breakdowns are obtained for the other 


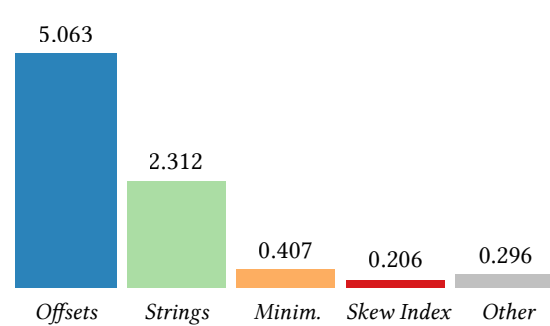

(a) regular -8.28 total bits/ $k$-mer

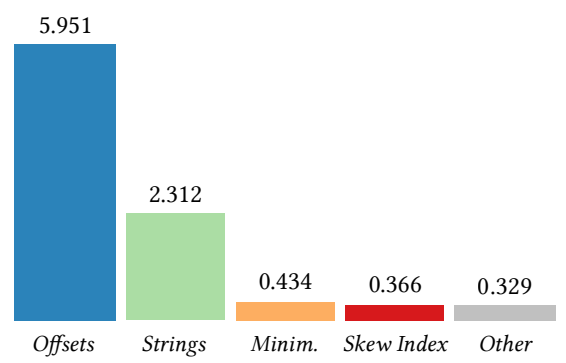

(b) canonical -9.39 total bits/ $k$-mer

Fig. 1. Space breakdowns for the Human dataset, for both regular (a) and canonical (b) dictionaries. The numbers above each bar indicate the bits/ $k$-mer spent by the respective components.

datasets. As expected, the most space-consuming component in the dictionary is the Offsets component (60-61\% of the total space). Hence, any effort to make SSHash more compact should be spent in making this component more light-weight. In this regard we recall that, because of the skew distribution of bucket size, most of the offsets' space is due to buckets of size 1: hence, Offsets is an array made by integers in the range $[0, N)$ essentially shuffled at random by the minimizers' MPHF. Therefore, our choice of spending $\left\lceil\log _{2} N\right\rceil$ bits per offset is basically optimal for such distribution. Lastly, the space difference between a regular and a canonical dictionary almost entirely resides in the Offsets component given that more minimizers are used, thus realizing a denser sampling.

The second most space-consuming component is Strings (28 - 32\% of the total space), which costs $2 N / n$ bits/ $k$-mer regardless of the parsing modality. While it could be possible to obtain better compression for Strings using a compressor for DNA (we only need to sequentially decompress a super- $k$-mer at lookup time) [Manzini and Rastero, 2004], we do not consider this as a promising option to explore given that most of the dictionary space is spent elsewhere (and given the slowdown in query processing that would follow). Both the MPHF on the minimizers (Minim.) and the skew index take a small fraction $(6-8 \%)$ of the total space. Other costs include those for representing the endpoints of the paths (Endpoints array) and size of the buckets (Sizes array): these costs are small, especially thanks to the use of the Elias-Fano encoding.

\subsection{Comparison Against Other Dictionaries}

In this section we compare SSHash against the following state-of-the-art dictionaries that we briefly reviewed in Section 3:

- dBG-FM [Chikhi et al., 2014] - An implementation of the popular FM-index [Ferragina and Manzini, 2000] tailored for DNA. This implementation is widely used as an exact membership data structure for $k$-mers [Chikhi et al., 2014, Rahman and Medvedev, 2020], also in the ABySS assembler [Simpson et al., 2009, Jackman et al., 2017].
Table 4. Dictionary space in total GB and average bits/ $k$-mer (bpk).

\begin{tabular}{|c|c|c|c|c|c|c|c|c|}
\hline \multirow{2}{*}{ Dictionary } & \multicolumn{2}{|c|}{ Cod } & \multicolumn{2}{|c|}{ Kestrel } & \multicolumn{2}{|c|}{ Human } & \multicolumn{2}{|c|}{ Bacterial } \\
\hline & GB & bpk & GB & bpk & GB & bpk & GB & bpk \\
\hline dBG-FM, $s=128$ & 0.22 & 3.48 & 0.44 & 3.07 & - & - & - & - \\
\hline dBG-FM, $s$ & 0.27 & 4.38 & 0.55 & 3.86 & - & - & - & - \\
\hline dBG-FM, $s=32$ & 0.39 & 6.16 & 0.78 & 5.43 & - & - & - & - \\
\hline \multirow[t]{2}{*}{ Pufferfish, sparse } & 1.75 & 27.80 & 3.69 & 25.66 & 8.87 & 28.32 & 18.91 & 28.28 \\
\hline & 1.49 & 23.70 & 3.37 & 23.40 & 7.50 & 23.96 & 16.09 & 24.06 \\
\hline \multirow[t]{2}{*}{ Pufferfish, dense } & 2.69 & 42.76 & 5.97 & 41.54 & 14.11 & 45.04 & 30.70 & 45.89 \\
\hline & 2.43 & 38.66 & 5.65 & 39.28 & 12.74 & 40.68 & 27.88 & 41.68 \\
\hline Blight & 0.91 & 14.53 & 2.16 & 15.00 & 5.04 & 16.11 & 11.40 & 17.04 \\
\hline Blight & 1.04 & 16.57 & 2.45 & 17.04 & 5.67 & 18.12 & 12.74 & 19.05 \\
\hline Blight, $b=0$ & 1.17 & 18.61 & 2.74 & 19.06 & 6.32 & 20.17 & 14.12 & 21.11 \\
\hline & 0.44 & 6.98 & 0.93 & 6.48 & 2.59 & 8.28 & 5.50 & 8.22 \\
\hline SSHash, canonical & 0.50 & 7.92 & 1.00 & 7.30 & 2.94 & 9.39 & 6.17 & 9.22 \\
\hline
\end{tabular}

We tested the index by sampling one position of the suffix-array every $s$ positions, for $s=32,64,128$

- Pufferfish [Almodaresi et al., 2018] - An index based on minimal perfect hashing and optimized for streaming membership queries. We test both the dense and sparse versions of the index. The sparse version was obtained with parameters $s=9$ and $e=4$ as used in the original paper.

- Blight [Marchet et al., 2021] - An associative index also based on minimal perfect hashing and minimizers. We test the index with sampling rate $b=0,2,4$ and minimizer length $m=10$ as suggested in the paper. We recall that a sampling rate of $b>0$ reduces the index space by $b$ bits/ $k$-mer at the expense of query time.

We use the $\mathrm{C}++$ implementations from the respective authors; links to the respective GitHub libraries are provided in the References. All sources were compiled using the same compilation flags as used for SSHash.

We first consider the space taken by the different tested dictionaries and reported in Table 4. The space of SSHash is significantly better than that of the other approaches based on minimal perfect hashing, roughly: $2-2.5 \times$ (or more) better than Blight, and $3-5 \times$ better than Pufferfish. This is so primarily because these approaches build a MPHF for the entire set of $k$-mers, hence associate a positional information (e.g., in the reference genome) to each $k$-mer in the input. We point out that, unlike for Blight, this is expected for Pufferfish dense since it was exactly designed for the purpose of reference mapping. (The shaded rows in Table 4 account for the space needed by Pufferfish to only support Lookup, i.e., discarding the color information in its colored de Bruijn graph structure.)

The dBG-FM index is, not surprisingly, the most compact ${ }^{2}$, thanks to the compression of the powerful Burrows-Wheeler transform (BWT) [Burrows and Wheeler, 1994]. While dBG-FM is several times smaller than Pufferfish and Blight, note that its smallest version tested (for $s=128$ ) is only essentially $2 \times$ smaller than regular SSHash and this gap diminishes at higher sampling rates. For example, dBG-FM for $s=32$ is only $13-17 \%$ smaller than regular SSHash. However, SSHash answers lookup queries much faster than dBG-FM as shown in Table 5. The lookup timings for $\mathrm{dBG}-\mathrm{FM}$ reported in the table were collected with the same methodology used for SSHash (see the beginning of Section 5.1). A lookup query in the dBG-FM index is implemented as a classic count query on a FM-index (see the paper by Ferragina and Manzini [2000] for details) which, for a pattern of length $k$, generates at least $k$ cache-misses. This cost

${ }^{2}$ We were unable to build the index correctly on the larger Human and Bacterial datasets. 
Table 5. Average Lookup time in ns/ $k$-mer for SSHash and the dBG-FM index.

\begin{tabular}{lrrrrr}
\hline \multirow{2}{*}{ Dictionary } & \multicolumn{2}{c}{ Cod } & & \multicolumn{2}{c}{ Kestrel } \\
\cline { 2 - 3 } & Lkp $^{+}$ & Lkp $^{-}$ & & Lkp $^{+}$ & Lkp $^{-}$ \\
\hline dBG-FM, $s=128$ & 22,980 & 16,501 & & 23,934 & 16,764 \\
dBG-FM, $s=64$ & 15,013 & 10,919 & & 15,929 & 11,462 \\
dBG-FM, $s=32$ & 11,386 & 7929 & & 11,703 & 8073 \\
\hline SSHash, regular & 1594 & 1158 & & 1042 & 1265 \\
SSHash, canonical & 834 & 690 & & 882 & 781 \\
\hline
\end{tabular}

is even higher for the handling of reverse complements that may induce two distinct count queries.

We also point out that the dBG-FM index needs to build the BWT of the input prior to indexing. This step can be very time consuming for large collections such as the ones of practical interest. That is, another important advantage of schemes based on minimal perfect hashing compared to BWT-based indexes is that they require significantly less time to build In this regard, we report that the construction time of SSHash, using a single processing thread, is very competitive with that of Pufferfish and Blight. For example, Blight takes 25 minutes to index the Human dataset with a single core, whereas SSHash takes just 13 minutes. However we remark that the construction of SSHash is not yet optimized to use multi-threading nor external memory. For this reason we do not explicitly compare construction times in this version of the work.

We now consider streaming membership queries to compare SSHash's query time to that of Pufferfish and Blight. Pufferfish and Blight are optimized to answer these kind of stateful queries, as exposed by their public software API. To query the dictionaries, we use some reads (in .fastq format) downloaded from the European Nucleotide Archive (ENA), and related to each dataset:

- for Cod: run accession SRR12858649 with 2,041,092 reads, each of length 110 bases;

- for Kestrel: run accession SRR11449743 with 14,647,106 reads, each of length 125 bases;

- for Human: run accession SRR5833294 with 34,129,891 reads, each of length 76 bases;

- for Bacterial: run accession SRR5901135 with 4,628,576 reads of variable length (a sequencing run of Escherichia Coli).

All dictionaries are fully loaded into memory before running the queries. We lookup for every $k$-mer read in sequence from the query files, adopting the same benchmark methodology used in both Pufferfish'sand Blight's papers. For the Human and Bacterial datasets we also use the same read as query files. For all the indexes, we just count the number of returned results rather than saving them to a vector. The result is reported in Table 6.

In general terms, we see that SSHash is either comparable to or faster (by $2-3 \times$ ) than Pufferfish and Blight. This holds true for both high-hit workloads ( $>70 \%$ hits, i.e., $k$-mers present in the dictionary) and low-hit workloads ( $<1 \%$ hits). It is important to benchmark the dictionaries under these two different query scenarios as both situations are meaningful in practice. (In our experiments, low-hit workloads are obtained by querying the dictionaries using a different query file as indicated in Table 6.) Indeed, observe that while Pufferfish's performance is robust under both scenarios, Blight's query time significantly degrades when most queries are negative, especially for $b>0$. Also regular SSHash is almost $2 \times$ slower for low-hit workloads compared to high-hit workloads. This is expected, however, because almost all queries are exhaustively inspecting two buckets per $k$ mer as we explained in Section 4.1. Note that its performance is anyway better than Blight's and not much worse than Pufferfish's (dense variant).
Table 6. Query time for streaming membership queries for various dictionaries. The query time is reported as total time in minutes (tot), and average ns/ $k$-me (avg). We also indicate the query file (SRR number) and the percentage of hits. Both high-hit ( $>70 \%$ hits) and low-hit ( $<1 \%$ hits) workloads are considered.

\begin{tabular}{|c|c|c|c|c|c|c|c|c|}
\hline \multirow{4}{*}{ Dictionary } & \multicolumn{2}{|c|}{ Cod } & \multicolumn{2}{|c|}{ Kestrel } & \multicolumn{2}{|c|}{ Human } & \multicolumn{2}{|c|}{ Bacterial } \\
\hline & \multirow{2}{*}{\multicolumn{2}{|c|}{$\begin{array}{c}\text { SRR } 12858649 \\
81.37 \% \text { hits }\end{array}$}} & \multirow{2}{*}{\multicolumn{2}{|c|}{$\begin{array}{c}\text { SRR11449743 } \\
74.60 \% \text { hits }\end{array}$}} & \multirow{2}{*}{\multicolumn{2}{|c|}{$\begin{array}{l}\text { SRR5833294 } \\
91.65 \% \text { hits }\end{array}$}} & \multirow{2}{*}{\multicolumn{2}{|c|}{$\begin{array}{l}\text { SRR5901135 } \\
87.79 \% \text { hits }\end{array}$}} \\
\hline & & & & & & & & \\
\hline & tot & avg & tot & avg & tot & avg & tot & avg \\
\hline Pufferfish, sparse & 0.6 & 214 & 14.1 & 609 & 17.0 & 651 & 9.1 & 691 \\
\hline Pufferfish, dense & 0.2 & 92 & 8.5 & 368 & 10.5 & 402 & 5.3 & 404 \\
\hline Blight, $b=4$ & 2.1 & 766 & 32.5 & 1400 & 27.3 & 1041 & 11.4 & 864 \\
\hline Blight, $b=2$ & 1.2 & 453 & 16.6 & 714 & 17.5 & 670 & 8.6 & 648 \\
\hline Blight, $b=0$ & 0.8 & 282 & 10.8 & 464 & 11.5 & 440 & 5.8 & 434 \\
\hline SSHash, regular & 0.5 & 166 & 6.2 & 267 & 8.2 & 311 & 3.0 & 223 \\
\hline SSHash, canonical & 0.3 & 111 & 5.1 & 219 & 6.7 & 253 & 2.4 & 184 \\
\hline
\end{tabular}

(a) high-hit workload

\begin{tabular}{|c|c|c|c|c|c|c|c|}
\hline \multirow{4}{*}{ Dictionary } & \multicolumn{2}{|c|}{ Cod } & \multicolumn{2}{|c|}{ Kestrel } & \multicolumn{2}{|c|}{ Human } & Bacterial \\
\hline & \multirow{2}{*}{\multicolumn{2}{|c|}{$\begin{array}{c}\text { SRR11449743 } \\
0.659 \% \text { hits }\end{array}$}} & \multirow{2}{*}{\multicolumn{2}{|c|}{$\begin{array}{c}\text { SRR12858649 } \\
0.484 \% \text { hits }\end{array}$}} & \multirow{2}{*}{\multicolumn{2}{|c|}{$\begin{array}{l}\text { SRR5901135 } \\
0.002 \% \text { hits }\end{array}$}} & \multirow{2}{*}{$\begin{array}{l}\text { SRR5833294 } \\
0.086 \% \text { hits }\end{array}$} \\
\hline & & & & & & & \\
\hline & tot & avg & tot & avg & tot & avg & tot avg \\
\hline Pufferfish, sparse & 14.6 & 627 & 0.9 & 312 & 11.3 & 855 & $25.5 \quad 975$ \\
\hline Pufferfish, dense & 8.7 & 374 & 0.2 & 92 & 5.8 & 435 & $13.6 \quad 518$ \\
\hline Blight, $b=4$ & 72.2 & 3112 & 6.6 & 2407 & 35.7 & 2704 & 253.29675 \\
\hline Blight, $b=2$ & 45.9 & 1978 & 3.0 & 1115 & 19.1 & 1445 & 117.74498 \\
\hline Blight, $b=0$ & 18.1 & 780 & 1.8 & 655 & 14.4 & 1088 & $32.2 \quad 1232$ \\
\hline SSHash, regular & 10.7 & 463 & 0.9 & 314 & 6.2 & 463 & $14.3 \quad 544$ \\
\hline SSHash, canonical & 5.1 & 220 & 0.4 & 155 & 2.5 & 183 & $6.4 \quad 244$ \\
\hline
\end{tabular}

(b) low-hit workload

The canonical version of SSHash protects against this behavior in case of low-hit workload and, in fact, is generally the fastest dictionary.

Another meaningful point to mention is that SSHash does not allocate extra memory at query time, i.e., only the memory of the index - as reported in Table 4 - is retained (the memory for the state information maintained by the streaming algorithm described in Section 4.3 is constant). Pufferfish also does not allocate extra memory. Blight, instead, consumes more memory at query time than that required by its index layout on disk. For example, to perform the queries on the Human dataset in Table 6a, Blight with $b=0$ uses a maximum resident set size of $7.51 \mathrm{~GB}$ compared to the $6.32 \mathrm{~GB}$ taken by its index on disk ( 23.98 vs. $20.17 \mathrm{bits} / k$-mer). This effect is even accentuated for higher $b$ values.

\section{Conclusions and Future Work}

We have studied the compressed dictionary problem for $k$-mers and proposed a solution, SSHash, based on a careful orchestration of minimal perfect hashing and compact encodings. In particular, SSHash is an exact and associative $k$-mer dictionary designed to deliver good practical performance. From a technical perspective, SSHash exploits the sparseness and the skew distribution of $k$-mer minimizers to achieve compact space, while allowing fast lookup queries.

We tested SSHash on collections of billions of $k$-mers and compared it against other indexes, under different query workloads (high- vs. low- hit) and modalities (random vs. streaming). Our implementation of SSHash is written in $\mathrm{C}++$ and open source. 
Compared to BWT-based indexes (like the dBG-FM index), SSHash is more than one order of magnitudes faster at lookup for only $2 \times$ larger space on average. Compared to prior schemes based on minimal perfect hashing (like Pufferfish and Blight), SSHash is significantly more compact (2 $5 \times$ depending on the configuration) without sacrificing query efficiency. Indeed, SSHash is also the fastest dictionary for streaming membership queries. For these reasons we believe that SSHash embodies a superior space/time trade-off for the problem tackled in this work.

Several avenues for future work are possible. We mention some promising ones. First, we will engineer the dictionary construction to use multi-threading and external memory. Parallel query processing is also interesting; since SSHash is a read-only data structure, its queries are amenable to parallelism. We could also add support for other types of queries, such as navigational queries [Chikhi et al., 2014]. Another promising direction could adapt the SSHash data structure to also store the abundances of $k$-mers, which is a separate but related problem in the literature [Shibuya et al., 2021, Italiano et al., 2021]. Based on the observation that consecutive $k$-mers tend to have the same or very similar abundance [Italiano et al., 2021], we expect to add a small extra space to SSHash to store this information. In this paper we focused on minimizers for their simplicity and practical efficiency but one could also explore the effects of replacing the minimizers with other types of string sampling mechanisms [Loukides and Pissis, 2021, Sahlin, 2021]. Lastly, we also plan to study the approximate version of the dictioanary problem where it is allowed to tolerate a prescribed false positive rate.

\section{Acknowledgments}

We thank Rossano Venturini for useful discussions on this work.

\section{Funding}

This work was partially supported by the projects: MobiDataLab (EU H2020 RIA, grant agreement № 101006879) and OK-INSAID (MIURPON 2018, grant agreement №ARS01_00917).

\section{References}

Fatemeh Almodaresi, Hirak Sarkar, Avi Srivastava, and Rob Patro. A space and time-efficient index for the compacted colored de bruijn graph. Bioinformatics, 34 (13):i169-i177, 2018. URL https://github.com/COMBINE-lab/pufferfish.

Austin Appleby. Smhasher. https://github.com/aappleby/smhasher, 2016.

Timo Bingmann, Phelim Bradley, Florian Gauger, and Zamin Iqbal. Cobs: a compact bit-sliced signature index. In International Symposium on String Processing and Information Retrieval, pages 285-303. Springer, 2019.

Michael Burrows and David Wheeler. A block-sorting lossless data compression algorithm. In Digital SRC Research Report. Citeseer, 1994.

Rayan Chikhi, Antoine Limasset, Shaun Jackman, Jared T Simpson, and Paul Medvedev. On the representation of de bruijn graphs. In International conference on Research in computational molecular biology, pages 35-55. Springer, 2014 URL https://github.com/jts/dbgfm.

Rayan Chikhi, Antoine Limasset, and Paul Medvedev. Compacting de bruijn graphs from sequencing data quickly and in low memory. Bioinformatics, 32(12):i201i208, 2016. URL https://github.com/GATB/bcalm.

Rayan Chikhi, Jan Holub, and Paul Medvedev. Data structures to represent a set of k-long dna sequences. ACM Computing Surveys (CSUR), 54(1):1-22, 2021

Peter Elias. Efficient storage and retrieval by content and address of static files Journal of the ACM, 21(2):246-260, 1974.

Robert Mario Fano. On the number of bits required to implement an associative memory. Memorandum 61, Computer Structures Group, MIT, 1971.
Paolo Ferragina and Giovanni Manzini. Opportunistic data structures with applications. In Proceedings 41st Annual Symposium on Foundations of Computer Science, pages 390-398. IEEE, 2000.

Guillaume Holley and Páll Melsted. Bifrost: highly parallel construction and indexing of colored and compacted de bruijn graphs. Genome biology, 21(1):1-20, 2020.

Giuseppe Italiano, Nicola Prezza, Blerina Sinaimeri, and Rossano Venturini. Compressed weighted de bruijn graphs. In 32nd Annual Symposium on Combinatorial Pattern Matching (CPM 2021), pages 1-16, 2021

Shaun D Jackman, Benjamin P Vandervalk, Hamid Mohamadi, Justin Chu, Sarah Yeo, S Austin Hammond, Golnaz Jahesh, Hamza Khan, Lauren Coombe, Rene L Warren, et al. Abyss 2.0: resource-efficient assembly of large genomes using a bloom filter. Genome research, 27(5):768-777, 2017

Jamshed Khan and Rob Patro. Cuttlefish: fast, parallel and low-memory compaction of de bruijn graphs from large-scale genome collections. Bioinformatics, 37 (Supplement_1):i177-i186, 2021

Jamshed Khan, Marek Kokot, Sebastian Deorowicz, and Rob Patro. Scalable, ultrafast, and low-memory construction of compacted de bruijn graphs with cuttlefish 2. bioRxiv, 2021

Yang Li, Pegah Kamousi, Fangqiu Han, Shengqi Yang, Xifeng Yan, and Subhash Suri. Memory efficient minimum substring partitioning. Proceedings of the VLDB Endowment, 6(3):169-180, 2013

Grigorios Loukides and Solon P Pissis. Bidirectional string anchors: A new strin sampling mechanism. In 29th Annual European Symposium on Algorithms (ESA 2021), pages 1-64, 2021

Giovanni Manzini and Marcella Rastero. A simple and fast DNA compressor. Softw Pract. Exp., 34(14):1397-1411, 2004. doi: 10.1002/spe.619.

Camille Marchet, Mael Kerbiriou, and Antoine Limasset. Blight: Efficient exact associative structure for k-mers. Bioinformatics, 2021. URL https://github.com/ Malfoy/Blight.

Miguel A Martínez-Prieto, Nieves Brisaboa, Rodrigo Cánovas, Francisco Claude, and Gonzalo Navarro. Practical compressed string dictionaries. Information Systems, 56:73-108, 2016.

Kurt Mehlhorn. On the program size of perfect and universal hash functions. In $23 \mathrm{rd}$ Annual Symposium on Foundations of Computer Science, pages 170-175. IEEE 1982

Giulio Ermanno Pibiri and Roberto Trani. Pthash: Revisiting FCH minimal perfect hashing. In SIGIR '21: The 44th International ACM SIGIR Conference on Research and Development in Information Retrieval, Virtual Event, Canada, July 11-15, 2021, pages 1339-1348. ACM, 2021a. doi: 10.1145/3404835.3462849.

Giulio Ermanno Pibiri and Roberto Trani. Parallel and external-memory construction of minimal perfect hash functions with pthash. CoRR, abs/2106.02350, 2021b URL https://arxiv.org/abs/2106.02350.

Amatur Rahman and Paul Medvedev. Representation of $k$-mer sets using spectrumpreserving string sets. In International Conference on Research in Computational Molecular Biology, pages 152-168. Springer, 2020. URL https://github.com/ medvedevgroup/UST.

Michael Roberts, Wayne Hayes, Brian R Hunt, Stephen M Mount, and James A Yorke. Reducing storage requirements for biological sequence comparison. Bioinformatics, 20(18):3363-3369, 2004

Lucas Robidou and Pierre Peterlongo. findere: Fast and precise approximate membership query. In String Processing and Information Retrieval, pages 151 163, Cham, 2021. Springer International Publishing. ISBN 978-3-030-86692-1.

Kristoffer Sahlin. Strobemers: an alternative to k-mers for sequence comparison. bioRxiv, 2021

Saul Schleimer, Daniel S Wilkerson, and Alex Aiken. Winnowing: local algorithm for document fingerprinting. In Proceedings of the 2003 ACM SIGMOD international conference on Management of data, pages 76-85, 2003.

Yoshihiro Shibuya, Djamal Belazzougui, and Gregory Kucherov. Space-efficient representation of genomic k-mer count tables. In 21 st International Workshop on Algorithms in Bioinformatics (WABI 2021), volume 201, pages 8-1, 2021.

Jared T Simpson, Kim Wong, Shaun D Jackman, Jacqueline E Schein, Steven JM Jones, and Inanç Birol. Abyss: a parallel assembler for short read sequence data. Genome research, 19(6):1117-1123, 2009.

Hongyu Zheng, Carl Kingsford, and Guillaume Marçais. Improved design an analysis of practical minimizers. Bioinformatics, 36(Supplement_1):i119-i127, 2020 . 